\title{
Computational Systems Biology as an Animal- Free Approach to Characterize Toxicological Effects of Persistent Organic Pollutants
}

\author{
Qier Wu, Rayane Achebouche and Karine Audouze \\ Université de Paris, Inserm UMR S-1124, Paris, France
}

\begin{abstract}
Exposure to persistent organic pollutants (POPs), as defined by the Stockholm Convention, may alter biological systems and cause toxic effects. Computational studies appear to be a relevant approach to increase our understanding of the molecular mechanisms triggered by POPs. We investigated the use of a systems toxicology approach to explore the effects of POPs on human health. A protein-protein association network (PPAN) was developed based on known POP-protein interactions. This model was used to predict protein complexes for several candidate POPs, including dicofol, methoxychlor, and perfluorooctanoic acid (PFOA), that are listed or proposed to be listed as POPs by the Stockholm Convention. Integration of multiple data sources (pathways, disease annotations, adverse outcome pathways) involving the identified protein complexes was performed independently in order to reveal putative risk factors for human health. This approach revealed that several systems may be disturbed by these candidate POPs, mainly the reproductive, metabolic and nervous systems.

This study highlights that a computational systems toxicology approach may help to decipher putative biological mechanisms of poorly studied chemicals and link them to possible adverse effects with the aim to support regulatory assessment and trigger new epidemiological and experimental studies. In order to develop more accurate computational models as alternative methods to animal testing, the next challenge will be to integrate more data according to the findable, accessible, interoperable and reusable (FAIR) data principles.
\end{abstract}

\section{Introduction}

Persistent organic pollutants (POPs) are organic chemicals that persist for long time periods in the environment and that are resistant to environmental degradation by chemical, biological and photolytic processes. Therefore, they are capable of long-range transport, bioaccumulation in human and animal tissues (e.g., adipose tissue), and bio-magnification in food chains. POPs include dioxin, polychlorinated biphenyls (PCBs), polybrominated diphenyl ethers (PBDEs), and hexabromocyclodecane (HBCD).

Exposure to such chemicals may have significant impacts on human health, animals as well as on the environment. Previous studies (from epidemiology to in vitro studies) have shown that some POPs may contribute to health disorders such as certain cancers, birth defects, dysfunctional immunity, reproductive and neurological disorders (Koual et al., 2019; Deng et al., 2019). With the aim of protecting human health and the environment, an international agreement, the Stockholm Convention on POPs ${ }^{1}$, was established and adopted in 2001, and is effective since May 2004. The main objectives of this convention are to eliminate or restrict the production and use of chemical substances defined as POPs and to reduce their unintentional release. In 2007, 16 substances were added to the 12 initial POPs covered by this convention ${ }^{2}$.

Besides taking measures to regulate the production and use of these chemicals, it is essential to gain a better understanding of their biological modes of action (MoA) and pathways of toxicity $(\mathrm{PoT})$ to understand their impact on human health (Kleensang et

\footnotetext{
1 http://www.pops.int/TheConvention/Overview/TextoftheConvention/tabid/2232/Default.aspx

2 http://chm.pops.int/tabid/3486/Default.aspx
}

Received October 16, 2019; Accepted January 14, 2020; Epub January 21, 2020; (C) The Authors, 2020

ALTEX 37(2), 287-299. doi:10.14573/altex.1910161

Correspondence: Karine Audouze, PhD

Université de Paris, Inserm UMR-S 1124

45 rue des Saints-Pères, 75006 Paris, France

(karine.audouze@univ-paris-diderot.fr)
This is an Open Access article distributed under the terms of the Creative Commons Attribution 4.0 International license (http://creativecommons.org/licenses/by/4.0/) which permits unrestricted use, distribution and reproduction in any medium, provided the original work is appropriately cited. 
al., 2014) and facilitate decision-making that supports regulation, innovation and competitiveness (Naderi et al., 2014). Further, the development of relevant, reliable and cost-effective methods can help to identify new substances with similar activities.

New approach methodologies (NAMs) are animal-free approaches that include computational models, in vitro high throughput screening (HTS), omics studies (Hartung et al., 2017), and the newly suggested $3 \mathrm{~S}$ approach (systematic, systemic, and systems biology and toxicology)(Smirnova et al., 2018). NAMs promise to capture a better understanding of the MoA of chemicals in humans and may help to support chemical safety assessment (Andersen et al., 2019). While they may not be able to completely substitute experimental studies, they can help to prioritize further experiments and thus support the $3 \mathrm{Rs}$ principle (Replacement, Reduction, Refinement).

During recent years, toxicological and chemical databases have expanded substantially, and the relevance of integrative systems toxicological methods has been recognized (Corton et al., 2019; Audouze and Grandjean, 2011; Audouze et al., 2013). The development of computational approaches for future toxicity assessment has been recommended by several committees including the U.S. EPA (Knudsen, 2013), the National Research Council (NRC, 2007; Krewski et al., 2010), the European Chemicals Agency (ECHA), the REACH legislation (Registration, Evaluation and Authorisation of CHemicals), and the Organization for Economic Co-operation and Development (OECD).

Many small molecules, including drugs and environmental chemicals, are known to affect multiple molecular targets and may deregulate proteins and, subsequently, functional pathways (Yildirim et al., 2007; Audouze et al., 2010). Here, we propose a computational model specific to the "biological space" of POPs to predict yet uncharacterized links between POPs and biological targets that may cause toxic effects. Since, in biological systems, proteins do not work as individual units but more in functional modules made up of two or more interacting proteins, we applied a computational network biology approach developed previously that associates protein pairs based on chemical-protein information (PPAN) (Audouze et al., 2010) to generate a computational systems toxicology model for persistent organic pollutants (POP-PPAN, POP-protein-protein association network).

This first global model, created using large sets of toxicogenomics data to explore the full biological space, was benchmarked against high confidence experimental protein-protein interactions, and predictions were validated by literature searches. This concept has previously been applied to other areas, with successful experimental validation of some chemical-protein predictions (Taboureau and Audouze, 2017; Audouze et al., 2014). The ability of the developed POP-PPAN model to predict new links by calculation of a binary score is demonstrated with several case studies, including dicofol and perfluorooctanoic acid (PFOA), compounds listed as POP candidates in May 2019 under the Stockholm Convention on POPs.

\section{Material and methods}

A workflow of the strategy to develop the computational systems toxicology model is shown in Figure 1. First, existing biological knowledge was extracted from the ToxCast and CompTox databases (Richard et al., 2016; Williams et al., 2017). Specific information on POP-protein interactions was compiled in order to create the network-based model POP-PPAN. Only pro-

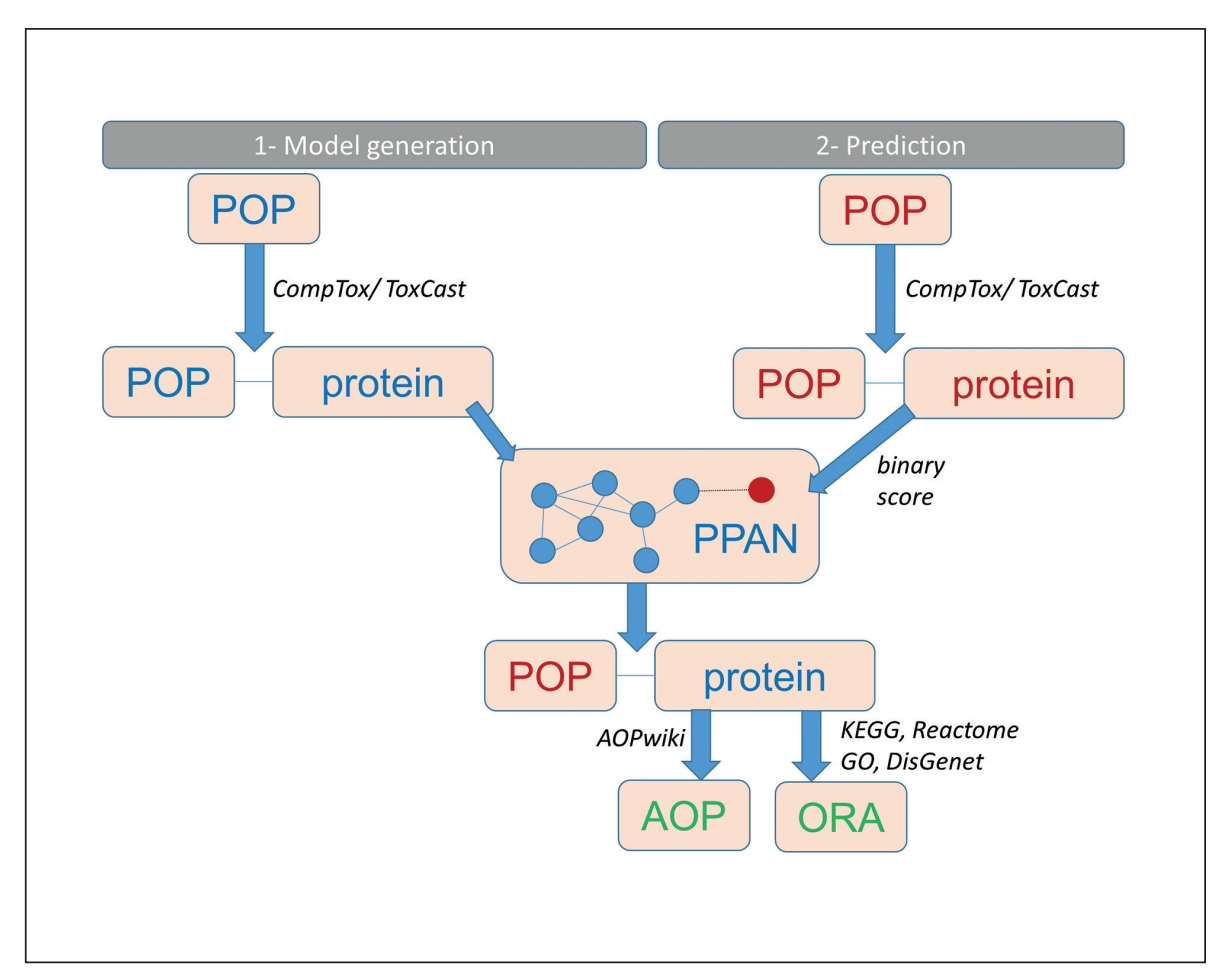

Fig. 1: Workflow of the computational systems toxicology approach to predict adverse effects of suspected persistent organic pollutants (POPs) 1-Model generation: Links between POPs from the Stockholm Convention list and proteins were extracted from the CompTox and ToxCast databases (in blue). The POP-PPAN model was created, based on a protein-protein association network procedure. 2-Prediction: Known proteins linked to suspected POPs (in red) were compiled. The developed model was then screened for these proteins; binary scores were calculated between known (red) and uncharacterized (blue) proteins for the suspected POP, using a neighbor protein procedure. Biological interpretation of the results was done after integration and over representation analysis (ORA) of various datasets (adverse outcome pathways (AOPs), pathways and diseases). 
Tab. 1: Chemicals currently listed as POPs under the Stockholm convention

For the compound classes (designated with an *), specific chemicals that are good representatives of these chemical families were selected, resulting in a total of 35 compounds.

\begin{tabular}{|c|c|c|c|c|c|}
\hline Inital POPs & CAS rn & & Newer POPs & CAS rn & \\
\hline Aldrin & $309-00-2$ & Insecticide & Alpha Hexachlorocyclohexane & $319-84-6$ & Insecticide \\
\hline Chlordane & $57-74-9$ & Pesticide & Beta Hexachlorocyclohexane & $319-85-7$ & Insecticide \\
\hline $\begin{array}{l}\text { Dichlorodiphenyltrichloroethane } \\
\text { (DDT) }\end{array}$ & $50-29-3$ & Pesticide & Chlordecone (=Kepone) & $143-50-0$ & Insecticide \\
\hline Dieldrin & $60-57-1$ & Insecticide & $\begin{array}{l}\text { Decabromodiphenyl ether } \\
\text { (DecaBDE = BDE 209) }\end{array}$ & $1163-19-5$ & Flame retardant \\
\hline Endrin & $72-20-8$ & Pesticide & Hexabromobiphenyl (PBB 153) & $36355-01-8$ & Flame retardant \\
\hline Heptachlor & $76-44-8$ & Pesticide & Hexabromocyclododecane & 25637-99-4 & Flame retardant \\
\hline Hexachlorobenzene (HCB) & $118-74-1$ & $\begin{array}{l}\text { Fungicide, } \\
\text { additive to } \\
\text { fireworks, } \\
\text { ammunition \& } \\
\text { synthetic rubber }\end{array}$ & Hexabromodiphenyl ether & $36483-60-0$ & \\
\hline Mirex & $2385-85-5$ & $\begin{array}{l}\text { Pesticide, } \\
\text { fire retardant }\end{array}$ & Heptabromodiphenyl ether & 68928-80-3 & \\
\hline Toxaphene & 8001-35-2 & Pesticide & Hexachlorobutadiene & 87-68-3 & \\
\hline Polychlorinated biohenyls (PCB)* & & $\begin{array}{l}\text { Insulating fluid; } \\
\text { paint; additive; } \\
\text { lubricant }\end{array}$ & Lindane & $58-89-9$ & Insecticide \\
\hline -2,2'-Dichlorobiphenyl & $13029-08-8$ & & Pentachlorobenzene & $608-93-5$ & Pesticide \\
\hline $\begin{array}{l}\text {-2,2',3,4',5,5',6-Heptachloro-1, } \\
\text { 1'-biphenyl }\end{array}$ & $52663-68-0$ & & Pentachlorophenol & $87-86-5$ & \\
\hline -2,2',4,4',5,5'-Hexachlorobiphenyl & $35065-27-1$ & & Perfluorooctane sulfonic acid (PFOS) & $1763-23-1$ & $\begin{array}{l}\text { Protective } \\
\text { coating }\end{array}$ \\
\hline $\begin{array}{l}\text { Polychlorinated dibenzo-p-dioxins } \\
(\mathrm{PCDD})^{\star}\end{array}$ & & Herbicide & $\begin{array}{l}\text { Perfluorooctane sulfonyl fluoride } \\
\text { (PFOSF) }\end{array}$ & $307-35-7$ & $\begin{array}{l}\text { Protective } \\
\text { coating }\end{array}$ \\
\hline $\begin{array}{l}\text {-2,3,7,8-Tetrachlorodibenzodioxin } \\
\text { (=TCDD) }\end{array}$ & $1746-01-6$ & & Pentabromodiphenyl ether & $32534-81-9$ & Flame retardant \\
\hline $\begin{array}{l}\text { Polychlorinated dibenzofurans } \\
(\text { PCDF) })^{*}\end{array}$ & & Herbicide & Tetrabromodiphenyl ether & $40088-47-9$ & \\
\hline - 2,3,4,7,8-Pentachlorodibenzofuran & $57117-31-4$ & & Polychlorinated naphthalenes* & & \\
\hline \multirow[t]{5}{*}{ - 1,2,3,7,8-Pentachlorodibenzofuran } & $57117-41-6$ & & -1-Chloronaphthalene & $90-13-1$ & \\
\hline & & & Endosulfan & $115-29-7$ & Pesticide \\
\hline & & & $\begin{array}{l}\text { Short chain chlorinated parafins } \\
\text { (SCCPs) }^{*}\end{array}$ & & \\
\hline & & & $\begin{array}{l}\text { - chlorinated paraffins: } \\
\text { C12, } 60 \% \text { chlorine }\end{array}$ & $\begin{array}{l}\text { no CAS rn } \\
\text { (PubChem } \\
\text { ID: } \\
5284361 \text { ) } \\
\end{array}$ & \\
\hline & & & $\begin{array}{l}\text { - 1,2,3,4,6,7,10- } \\
\text { Heptachlorododecane }\end{array}$ & $\begin{array}{l}1005111- \\
47-6\end{array}$ & \\
\hline
\end{tabular}

teins for which POPs are defined as "active" and with activity below their cytotoxicity threshold were included. A neighbor protein procedure was developed and applied, based on the binary interaction method used in high-throughput experiments to develop a scoring system that allows the prediction of uncharacterized links between 28 POP candidates (compounds listed or under review in the Stockholm Convention or identified from literature studies) and proteins. As a last step, biological information (adverse outcome pathways (AOPs), pathways, gene ontology terms (GO) and diseases) was integrated to identify statistically significant connections between the chemicals and the biological events. 


\section{Tab. 2: List of the 28 chemicals defined as POP candidates}

Chemicals that have proteins associated to them in the ToxCast database considering the cytotoxicity threshold are shown in grey. Chemicals that have more than 4 proteins associated to them, for which we performed an ORA analysis, are in bold font. NA, not analyzed for enrichment (proteins $<4$ )

\begin{tabular}{|c|c|c|c|c|c|}
\hline \multicolumn{2}{|l|}{ Chemical name } & \multirow{2}{*}{$\begin{array}{l}\text { CAS rn } \\
95-50-1\end{array}$} & \multirow{2}{*}{\begin{tabular}{|l|} 
Cytotoxicity \\
$1000 \mu \mathrm{M}$ \\
\end{tabular}} & \multirow{2}{*}{\begin{tabular}{|l}
$\begin{array}{l}\text { Number of } \\
\text { proteins from } \\
\text { assays }\end{array}$ \\
7 \\
\end{tabular}} & \multirow{2}{*}{\begin{tabular}{|l}
$\begin{array}{l}\text { Number of } \\
\text { proteins with } \\
\text { PPAN }\end{array}$ \\
18 \\
\end{tabular}} \\
\hline & 1,2-Dichlorobenzene & & & & \\
\hline BDE-47 & 2,2',4,4'-Tetrabromodiphenyl ether & $5436-43-1$ & $8.36 \mu \mathrm{M}$ & $\begin{array}{l}\text { no assays after } \\
\text { cytotox threshold }\end{array}$ & NA \\
\hline BDE-99 & 2,2',4,4',5-Pentabromodiphenyl ether & $60348-60-9$ & $6.94 \mu \mathrm{M}$ & $\begin{array}{l}\text { no assays after } \\
\text { cytotox threshold }\end{array}$ & NA \\
\hline BDE-153 & 2,2',4,4',5,5'-Hexabromodiphenyl ether & $68631-49-2$ & $6.66 \mu \mathrm{M}$ & $\begin{array}{l}\text { no assays after } \\
\text { cytotox threshold }\end{array}$ & NA \\
\hline \multirow[t]{4}{*}{ PCB 118} & 2,3',4,4',5-Pentachlorobiphenyl & $31508-00-6$ & no data & - & NA \\
\hline & 2,6-Dinitrotoluene & $606-20-2$ & $1000 \mu \mathrm{M}$ & 4 & NA \\
\hline & Atrazine & $1912-24-9$ & $1.25 \mu \mathrm{M}$ & 1 & NA \\
\hline & Benzene & $71-43-2$ & $1000 \mu \mathrm{M}$ & - & NA \\
\hline$\beta \mathrm{HCH}$ & beta-Hexachlorocyclohexane & $319-85-7$ & $11.38 \mu \mathrm{M}$ & 6 & 16 \\
\hline \multirow[t]{6}{*}{ Dechlorane Plus } & Bis(hexachlorocyclopentadieno)cyclooctane & $13560-89-9$ & no data & - & NA \\
\hline & Bisphenol-A & $80-05-7$ & $11.69 \mu \mathrm{M}$ & 33 & 39 \\
\hline & Chloramphenicol & $56-75-7$ & $1000 \mu \mathrm{M}$ & 4 & NA \\
\hline & Chlordimeform & $6164-98-3$ & $1000 \mu \mathrm{M}$ & 1 & NA \\
\hline & Chlorobenzene & $108-90-7$ & $1000 \mu \mathrm{M}$ & 1 & NA \\
\hline & Diazinon & $333-41-5$ & $1000 \mu \mathrm{M}$ & 30 & 35 \\
\hline \multirow[t]{6}{*}{ p,p'-DDE } & Dichlorodiphenyldichloroethylene & $72-55-9$ & $8.74 \mu \mathrm{M}$ & 4 & NA \\
\hline & Dimethomorph & $110488-70-5$ & $5.86 \mu \mathrm{M}$ & 13 & 21 \\
\hline & Methoxychlor & $72-43-5$ & $8.26 \mu \mathrm{M}$ & 11 & 18 \\
\hline & Naproxen & $22204-53-1$ & $1000 \mu \mathrm{M}$ & 0 & NA \\
\hline & Nitrobenzene & $98-95-3$ & $1000 \mu \mathrm{M}$ & 3 & NA \\
\hline & Parathion & $56-38-2$ & $8.74 \mu \mathrm{M}$ & 27 & 33 \\
\hline \multirow[t]{6}{*}{ PFHxS } & Perfluorohexane sulfonic acid & $355-46-4$ & no data & - & NA \\
\hline & Phenanthrene & 85-01-8 & $8.76 \mu \mathrm{M}$ & 3 & NA \\
\hline & Phenol & $108-95-2$ & $1000 \mu \mathrm{M}$ & 3 & NA \\
\hline & Prometryne & $7287-19-6$ & $8.28 \mu \mathrm{M}$ & 16 & 25 \\
\hline & Pyrene & $129-00-0$ & $11.46 \mu \mathrm{M}$ & 3 & NA \\
\hline & Pyrimethanil & $53112-28-0$ & $1000 \mu \mathrm{M}$ & 34 & 41 \\
\hline \multirow[t]{2}{*}{ PFOA } & Perfluorooctanoic acid & $335-67-1$ & $5.35 \mu \mathrm{M}$ & 9 & 17 \\
\hline & Dicofol & $115-32-2$ & $7.89 \mu \mathrm{M}$ & 18 & 28 \\
\hline
\end{tabular}

\subsection{Chemical data}

\section{Model generation}

We extracted the chemical names of the 28 POPs from the Stockholm Convention list (as of January 2019) ${ }^{3}$, i.e., the 12 initial POPs and the 16 new POPs (Tab. 1). The 12 initial POPs are chemicals that have been recognized as causing adverse effects on humans and the ecosystem. They are categorized into three classes, i.e., pesticides, industrial chemicals, and by-products. The 16 new chemicals are compounds with a risk profile; they are categorized in the same way as the 12 initial POPs.

\section{Prediction}

A list of 28 chemicals suspected to be POPs or semi-persistent organic pollutants (sPOPs) was established, based on data from

\footnotetext{
3 http://chm.pops.int/TheConvention/ThePOPs/tabid/673/Default.aspx
} 
the literature, chemicals recommended for listing by the review committee of the Stockholm Convention, and chemicals under review by the Stockholm Convention (Tab. 2).

\subsection{Chemical-protein associations}

Chemical-protein associations were extracted from the publicly available CompTox and ToxCast databases (last accessed November 2019). The CompTox database (Williams et al., 2017), an online database maintained by the U.S. Environmental Protection Agency (EPA), contains information regarding multiple data types (physico-chemical properties, exposure, biological activities, etc.) for over 87,500 chemicals. The CompTox dashboard was used to identify the biological assays in which the POPs and sPOPs are active to determine the cytotoxicity information and respective links to AOPs. The ToxCast database is a U.S. EPA program that builds large collections of endpoint data of more than 700 high-throughput assays on chemicals to which humans are potentially exposed (Richard et al., 2016; Kavlock et al., 2012). The ToxCast dashboard was used to obtain information about the assays extracted from the CompTox database, for example the protein identifiers.

For the current investigation, only proteins passing the filtering process using the "cytotoxic-associated burst" region were retained (Judson et al., 2016) (Tab. S14), as it has been demonstrated that compounds activate assays at concentration levels also observed for cytotoxicity or cell stress (Judson et al., 2016). Therefore, only assay activities (AC50) that occurred at a concentration below the "cytotoxic-associated burst" region were extracted.

\subsection{Generating a high-confidence protein-protein associations network model}

The relevant POP-protein links compiled and filtered from the CompTox and ToxCast databases were used to create the model. The POP-PPAN model was generated based on the previously published protein-protein association network (PPAN) approach (Audouze et al., 2010). Each protein (that linked to at least one POP) was represented as a node, and any protein-protein pairs for which at least one overlapping chemical was identified were linked by an edge. These protein-protein associations were converted into a non-redundant list of associations, i.e., if proteins $\mathrm{A}$ and $\mathrm{B}$ are linked, the network may have two associations A-B and B-A. In our approach, only one of these protein pairs was retained to create the model. To reduce noise and retain only the most relevant associations, each protein pair was scored (oS) based on the chemical overlapping information, i.e., counting shared POPs between each protein pair.

\subsection{Neighbor protein procedure}

To predict novel links between sPOPs and proteins, a neighbor protein procedure was developed, which is a three-step procedure: First, input proteins known to interact with the studied chemical were identified from the CompTox and ToxCast databases. Then, the developed POP-PPAN model was screened

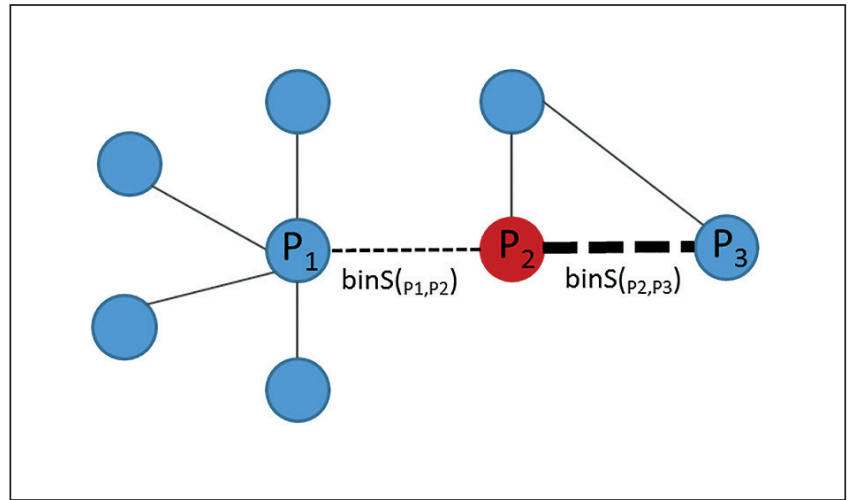

Fig. 2: Predictions of protein associations using a binary score (bins)

An input protein (in red) known to interact with a studied chemical is screened into the POP-PPAN model. As results, network of first order interacting proteins are identified (in blue), and binS are calculated to rank and select high-confidence interactions. The binS ( $\left.1, \mathrm{P}_{2}\right)$ is a low-confidence score, as it has six unshared interaction partners (all the other blue ones). The binS(P2, P3) has a higher confidence score as it has only one unshared interaction partner, i.e., P1. Therefore, proteins $\mathrm{P} 2$ and $\mathrm{P} 3$ are more likely to be associated in the POP space, and protein $\mathrm{P} 3$ is predicted as a potential target for the chemical of interest.

for the input proteins in order to determine the first-order interacting proteins and the surrounding networks. This established a network of interacting proteins that contains the input proteins and proteins from the model. The last step was to calculate a binary score (binS) between each pair of proteins to rank and select putative proteins for the sPOPs. The developed binS is based on quality scoring of protein-protein interactions (PPIs) used in high-throughput experiments (such as yeast two-hybrid and protein-fragment complementation assay) to determine the reliability of the PPIs in large-scale datasets (Yu et al., 2008; Tarassov et al., 2008). A previous study has shown that the reliability of interactions between proteins correlates well with the number of non-shared interaction proteins for each interacting pair (de Lichtenberg et al., 2005). A binS was calculated for each protein pair as follows:

$$
\operatorname{binS}(\mathrm{P} 1, \mathrm{P} 2)=-\log 10\left(\left(\mathrm{~N}_{\mathrm{P} 1}+1\right)\left(\mathrm{N}_{\mathrm{P} 2}+1\right)\right),
$$

where $N_{P 1}$ and $N_{P 2}$ are the numbers of non-shared interaction partners for an association between proteins $1(\mathrm{P} 1)$ and $2(\mathrm{P} 2)$ (Fig. 2).

\subsection{AOP-protein linkages}

To characterize AOPs connected to the protein complexes, AOP information was integrated into the developed model using data from the CompTox and AOP-wiki databases ${ }^{5}$ (August 28, 2019). In order to cover the biggest toxicological space possible,

\footnotetext{
4 doi:10.14573/altex.1910161s

5 https://aopwiki.org/
} 
and since few AOPs had been validated by the date of the study, AOPs under development were also integrated. The AOP-wiki database, which is part of a knowledge structure of AOPs (Villeneuve et al., 2014), is an online database based on a collaborative program between the OECD and the European Commission.

\subsection{Biological enrichment of the protein networks}

The protein complexes associated with the sPOPs were independently tested for biological significance and disease associations. All proteins were converted to EntrezGeneID to facilitate analysis. To assess the relevance of protein -GO, -pathway and -disease linkages, each data source was individually tested using an over-representation analysis (ORA) based on a hypergeometric distribution. A significance level of 0.05 after Benjamini-Hochberg correction for multiple testing of $p$-values was used to select the most relevant associations.

\section{Gene ontology-protein associations}

Investigation in terms of biological relevance was done for each of the GO categories, i.e., biological process, molecular function, and cellular component (Ashburner et al., 2000; The Gene Ontology Consortium, 2019).

\section{Pathway-protein associations}

Functional pathways that may potentially be disturbed by chemicals were investigated using the Kyoto Encyclopedia of Genes and Genomes (KEGG) and the Reactome database (Kanehisa et al., 2019; Fabregat et al., 2018) (last access November, 2019).

\section{Disease-protein enrichment}

For disease enrichment, the DisGeNET data source, which contains human protein-disease annotations, was used to identify disorders potentially connected with the studied chemicals (Piñero et al., 2017) (last access November, 2019). DisGenNET is the largest publicly available platform integrating knowledge on gene-disease linkages from diverse databases and published articles. The current version (v6.0) contains 628,685 gene-disease associations between 17,549 genes and 24,166 diseases and abnormal human phenotypes.

\section{Results}

Based on the CompTox and ToxCast databases, a PPAN model representative of the POP biological space was generated in order to predict potential toxic effects of suspected POPs (sPOPs).

We extracted the 28 POPs from the Stockholm Convention list. Among them, five are families of compounds; therefore, we selected specific chemicals that are good representatives of these chemical families to facilitate further analysis, resulting in a total of 35 compounds used to create the PPAN model (Tab. 1). Protein information could only be retrieved for 20 chemicals when their cytotoxicity thresholds, as defined by Judson et al. (2016), were taken into consideration ( Tab. $\mathrm{S1}^{4}$ ). The cytotoxicity thresholds allow distinction between toxicity due to the disruption of a spe- cific biomolecular function and toxicity due to general cell stress. These 20 chemicals were linked to 95 unique proteins via 247 associations; chlordecone was associated with 56 proteins (via 97 assays), and the human estrogen receptor 1 (ESR1) was associated with 10 compounds (via 29 assays) (Tab. S1 ${ }^{4}$ ). We were not able to retrieve bioactive records for hexachlorobenzene (HCB), hexabromobiphenyl (PBB 153), hexabromocyclododecane (HB$\mathrm{CD}$ ), and some polybrominated diphenyl ethers (PBDEs). To build the model, 198 unique associations between the 20 chemicals and 95 proteins were used.

\subsection{Generating a high-confidence protein-protein association network (PPAN) in the biological space of POPs}

Using the compiled chemical-protein information, we constructed a POP-PPAN model following the procedure developed previously (Audouze et al., 2010). This procedure is based on the assumption that if two proteins are biologically affected by the same POPs (defined as shared compounds), they are likely to be involved in a common mechanism of action of the chemicals. Therefore, two biological targets are linked to each other if they are associated with at least one common POP. The resulting model consists of 47 associations between 18 proteins (Fig. 3, Tab. $\mathrm{S} 2{ }^{4}$ ), meaning that many of the extracted proteins are not, or are not yet known to be, targeted by common POPs.

To highlight the most significant associations, an overlapping score $(\mathrm{oS})$ was assigned to each pair of proteins based on the calculation of overlapping POPs for each protein pair. The smallest oS value being 3 (for example, ESR1 and ESR2 are targeted by the 3 POPs bHCH, chlordane, and chlordecone); the highest score was 8 (ESR1 and NR1I2 are targeted by endosulfan, dieldrin, bHCH, endrin, DDT, chlordane, chlordecone, and aldrin).

In order to evaluate the POP-PPAN in the toxicological space, we went one step further and integrated biological events from the known AOPs into the protein complex network using both the CompTox and AOP-wiki databases. We were able to link 7 of the 18 proteins to 12 MIEs and 7 KEs (the event ID 122 is defined as a MIE and a KE in AOPwiki) that are involved in 21 different AOPs, mainly ones related to reproductive dysfunctions (see Tab. S3 ${ }^{4}$ for full names of the AOP events, and links to MIEs and KEs). Only one AOP (AOP 167, Early-life estrogen receptor activity leading to endometrial carcinoma in the mouse) is connected to two events (MIE 1064, Pre-pubertal increase, estrogen receptor (ER) activity and KE 1065, Activation, estrogen receptor alpha). Four MIEs are connected to two AOPs each, i.e., MIE 111 (Agonism, estrogen receptor), MIE 122 (Activation, glucocorticoid receptor), MIE 245 (Activation, PXR/ SXR), and MIE 1396 (Increased, glucocorticoid receptor activity) linked respectively to AOPs 29/52 (Estrogen receptor agonism leading to reproductive dysfunction / ER agonism leading to skewed sex ratios due to altered sexual differentiation in males), AOPs 14/214 (Glucocorticoid Receptor Activation Leading to Increased Disease Susceptibility / Network of SSRIs (selective serotonin reuptake inhibitors), AOPs 11/60 (Pentachlorophenol Acute Response by Percellome / PXR activation leading to hepatic steatosis), and AOPs 221/222 (Mental stress to depression / Mental stress to agitation). 


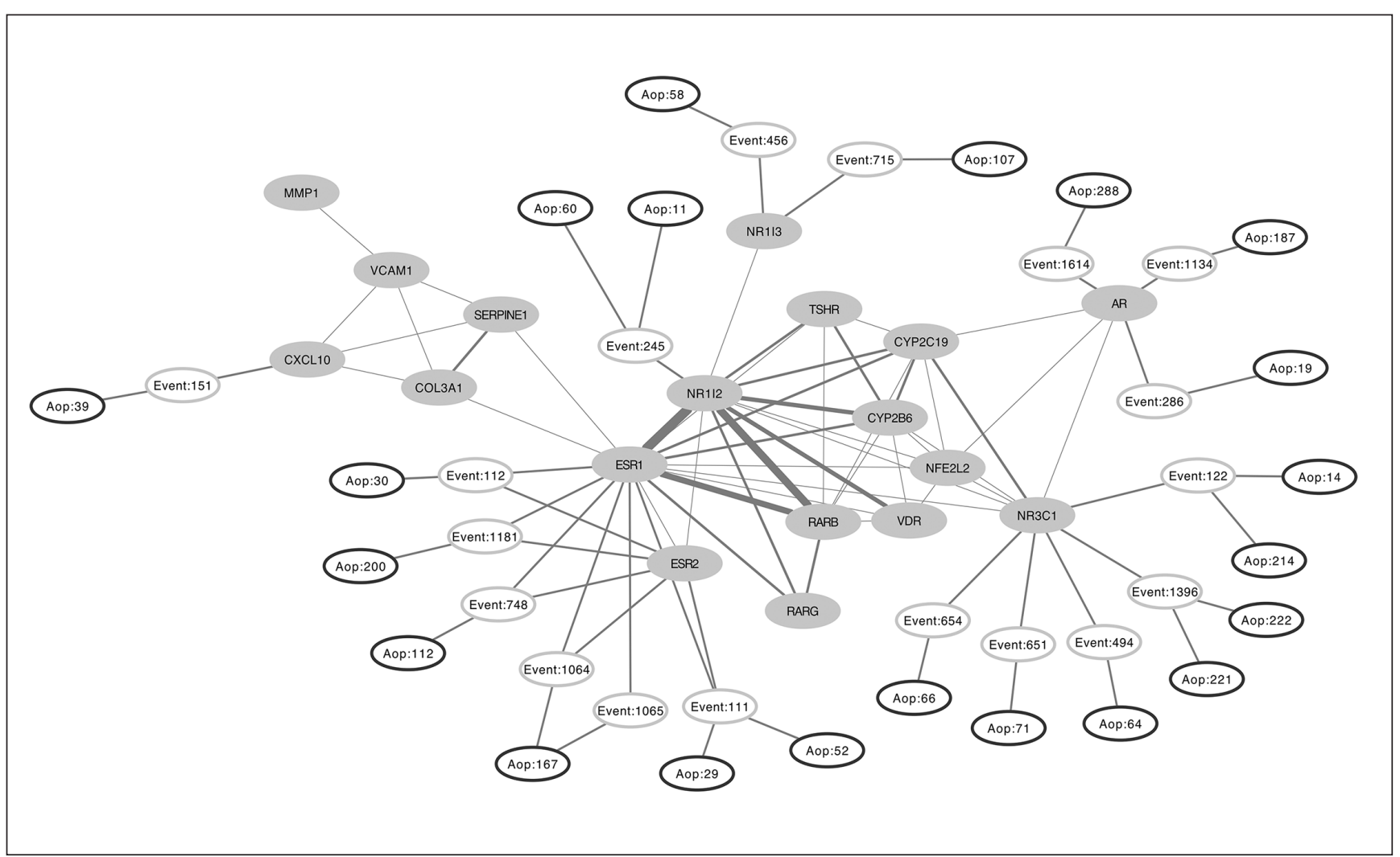

Fig. 3: Network representation of the top interactions from the POP-PPAN model in the AOP space

An edge is placed between two proteins (grey nodes) if they share at least one chemical (POP), and their width is proportional to the number of chemicals that are linked to both proteins (based on the calculated overlapping score (oS)). For example, the ESR1 and RARB share more POPS $(O S=6)$ than RARB and RARG $(O S=4)$. The white nodes circled in grey correspond to the biological events, and the white nodes circled in black to the AOPs extracted from the CompTox and AOP-wiki databases (for both, edges represent only a known interaction). The ID numbering is from the AOP-wiki database.

ESR1 is the protein connected to the most biological events (6), and ESR1 and ESR2 share events, reflecting that estrogen receptors are actively studied targets, probably due to their endocrine related activities.

\subsection{Using the POP-PPAN model for chemical toxicity prediction}

Besides revealing POP-AOP linkages, the network can be used to predict potential MoA for environmental chemicals. Our assumption is that if two proteins are affected by two chemicals, these proteins are associated in the chemical space. If one of the proteins is further deregulated by an additional chemical, then this compound may also deregulate the second protein. A hypothesis on how chemicals may affect human health can be based on a neighbor protein procedure. The binary score (binS) was developed based on the quality scoring of protein-protein interaction data used to evaluate the large-scale experimental data set. This allows us to calculate a score for each pair of proteins, to scan the POP-PPAN for each of the sPOPs present in the list, and therefore to predict relevant high-scoring associations between an sPOP and proteins. This neighbor protein procedure was applied to all 28 sPOPs; detailed analyses are presented for dicofol and PFOA below.

\subsubsection{Systems toxicology prediction of dicofol}

Dicofol is an organochlorine pesticide, structurally related to DDT, that is known to be involved in human adverse effects such as reproductive and neurobehavioral outcomes (Audouze and Grandjean, 2011). Dicofol is used as a pesticide and acaricide on a variety of fruits, vegetables, and field crops in many countries around the world. In May 2019, it was added to the Stockholm Convention on POPs due to its ability to bioaccumulate in living organisms and evidence of persistent criteria (half-life of 85 days in water and of 313 days in aerobic soil) (Kelly et al., $\left.2007 ;^{6}\right)$. Moreover, this substance has a high potential for longrange environmental transport, for example, it has been detected in the Artic environment (Zhong et al., 2012). Even if the MoA of dicofol are not yet fully identified, adverse effects on human health and other living organisms have been reported, including effects on liver, kidney, and adrenal glands (Liu and Liu, 2012).

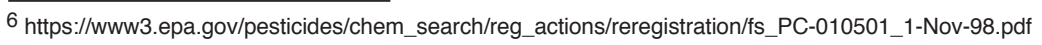




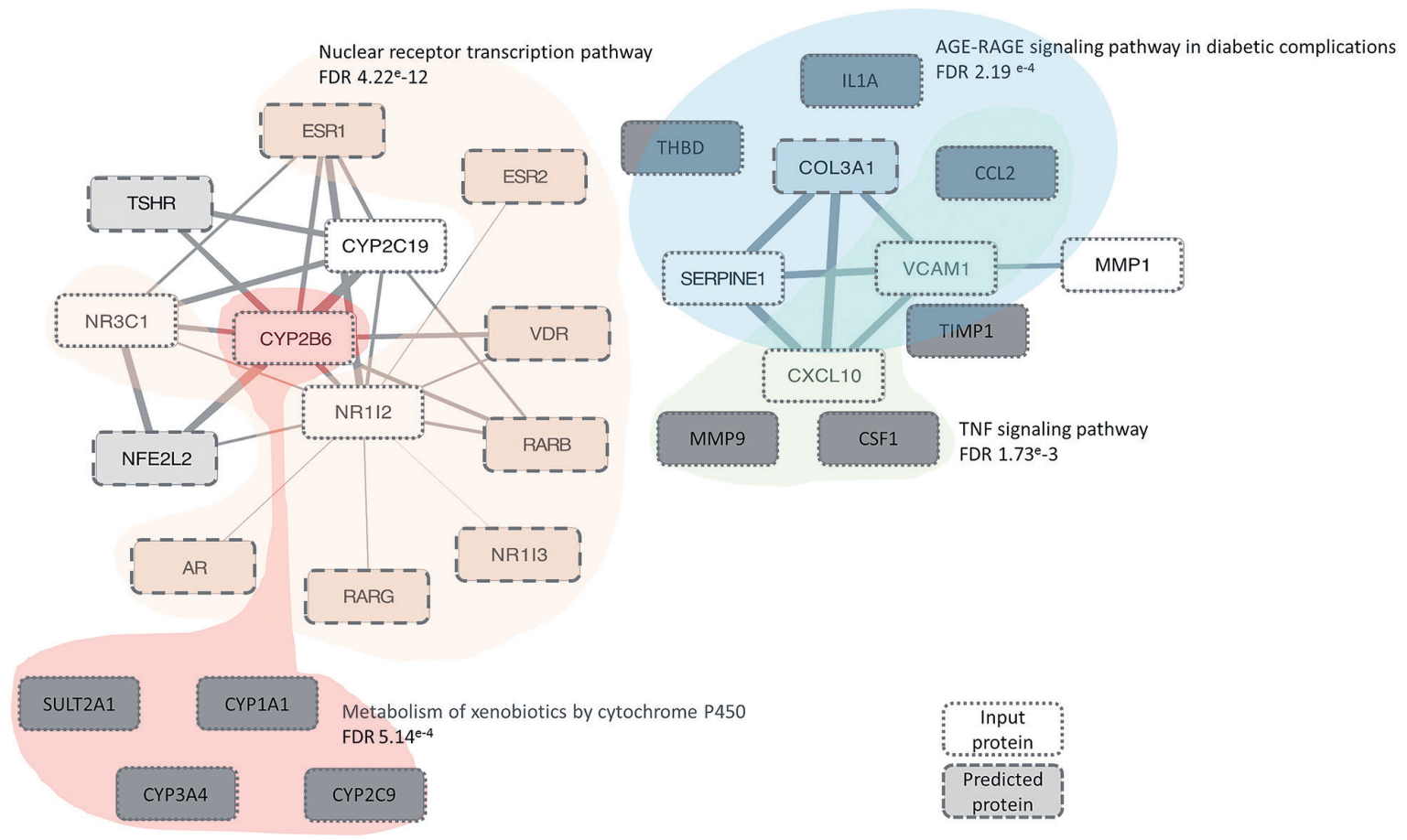

Fig. 4: Protein-protein association network for Dicofol in the POP space

Each node represents a protein, the input proteins being the ones extracted from the CompTox database, to which associated proteins from the POP-PPAN model were added (predicted proteins). The width of edges is according to the binary score (binS) calculated for each protein pair. Colored forms represent some significant pathways annotations from the KEGG and Reactome databases based on an overrepresentation analysis.

Dicofol may disturb biological functions, some being related to endocrine disruption effects (Vinggaard et al., 2000), and has been shown to exert generational perturbations (MacLellan et al., 1996). The World Health Organization (WHO) classifies dicofol as a Level II, "moderately hazardous" pesticide; the US EPA classifies dicofol in Group C, as "possible human carcinogen". Based on these concerns, we decided to explore the potential toxicity of dicofol using the developed POP-PPAN model in order to propose potential linkages between this substance and adverse health effects.

Proteins known to be deregulated by dicofol were extracted from the CompTox database (as of July 17, 2019). Considering the cytotoxicity threshold $(7.89 \mu \mathrm{M}$, CompTox database as of July 2019), we were able to identify 18 biological targets (Tab. S4 ${ }^{4}$ ). The POP-PPAN model was scanned with these 18 proteins, using the neighbor protein procedure. As a result, a protein complex of 18 proteins was identified that includes 10 proteins predicted to be associated with 8 of the known proteins, meaning that 10 proteins known to be linked to dicofol were not present in the protein complex (Tab. $\mathrm{S}^{4}$ ). The resulting protein complex for dicofol, which contains 33 protein-protein associations (PPAs), was connected to 9 biological KEs of the know targets and to 9 KEs of the predicted proteins, linking respectively to 9 and 12 AOPs
(Tab. S4 ${ }^{4}$ ). Most of the AOPs identified are linked to the reproductive and the nervous systems. Interestingly, the predicted glucocorticoid receptor NR3C1 (nuclear receptor subfamily 3 group $\mathrm{C}$ member 1) was associated with 7 AOPs, among these 3 are involved in male fertility dysfunctions and 3 in stress. A recent study from Nordkap et al. (2017) suggested a link between stress and testicular function that may be modulated by NR3C1, which is expressed in human testis (Chihara et al., 2016).

To obtain an overview of the pathways that may be dysregulated by the identified proteins, an ORA was performed on the full protein complex related to dicofol using the KEGG and Reactome databases (Fig. 4, Tab. S5 ${ }^{4}$ ). The KEGG analysis reveals that some proteins are involved in 20 pathways, and among them 12 were statistically significantly associated with proteins (false discovery rate $($ FDR $)>5 \%)$. The most significant KEGG pathway was AGE-RAGE signaling pathway in diabetic complications (FDR 2.19 -04, via CCL2; COL3A1; IL1A; SERPINE1; THBD; VCAM1), a well-studied cascade of signaling mechanisms that initiates metabolic disorders such as diabetic complications (Kay et al., 2016). Among the other KEGG pathways, several were associated with metabolism (metabolism of xenobiotics by cytochrome P450, FDR 5.14 ${ }^{\mathrm{e}-4}$ via CYP1A1; CYP2B6; CYP2C9; CYP3A4; SULT2A1; and linoleic acid metabolism, 
FDR 4.65e-3 via CYP2C19; CYP2C9; CYP3A4). Another significantly associated pathway was the signaling pathway of TNF (tumor necrosis factor), which is a multifunctional proinflammatory cytokine with effects on lipid metabolism, coagulation, insulin resistance, and endothelial function (FDR $1.73^{\mathrm{e}-3}$ via CCL2; CSF1; CXCL10; MMP9; VCAM1). The Reactome main pathway analysis $\left(\mathrm{Tab} . \mathrm{S}^{4}\right)$ further indicates that 9 of the 28 proteins are annotated to the nuclear receptor transcription pathway (FDR 4.22 ${ }^{\mathrm{e}-12}$, AR; ESR1; ESR2; NR1I2; NR1I3; NR3C1; RARB; RARG; VDR). In total, 20 significant pathways were identified with the Reactome ORA analysis, most of them involving the different cytochromes associated with dicofol.

The enrichment analysis performed with the GO terms revealed that 27 of the 28 proteins are involved in metabolic processes (GO:0008152) and 10 are linked to reproduction (GO:0000003) (all biological processes, molecular functions, and cellular components are shown in Tab. $\mathrm{S}^{4}$ ).

As a last step, disease enrichment was performed on the identified protein complex using the DisGeNET database. This allows us to highlight potential links between dicofol and disorders of the nervous system (depressive symptoms, unipolar depression, major depressive disorder, and brain ischemia with respective FDRs of $1.11^{\mathrm{e}-6}, 3.22^{\mathrm{e}-3}, 3.40^{\mathrm{e}-3}$, and 0.03$)$ and to the reproductive system (prostatic neoplasms, mammary neoplasms, female infertility, prostatic intraepithelial neoplasias, and endometrial neoplasm, with respective FDRs of $1.15^{\mathrm{e}-5}, 3.88^{\mathrm{e}-4}, 7.98^{\mathrm{e}-4}$, $1.65^{\mathrm{e}-3}$, and $\left.4.98^{\mathrm{e}-3}\right)\left(\mathrm{Tab} . \mathrm{S} 5^{4}\right)$. Moreover, linkages were found for metabolic syndrome $\mathrm{X}$, which is a cluster of conditions that occur together, leading to increased risk of heart disease, stroke, and type 2 diabetes as well as diabetic nephropathy, which is kidney damage that results from having diabetes.

\subsubsection{Systems toxicology prediction of perfluorooctanoic acid (PFOA)}

PFOA and its related substances are widely used in applications and consumer products such as fire-fighting foams, wetting agents, and cleaners. PFOA can be found in textiles, food packaging, and various paints to mention but a few sources. This chemical is highly persistent and does not undergo any further abiotic or biotic degradation under environmental conditions owing to its hydrolytically stable properties. Moreover, studies have shown that PFOA bio-accumulates in humans (Haug et al., 2010, 2011), and experimental and epidemiological evidence shows that PFOA is linked to adverse effects in humans and wildlife, e.g., affecting the endocrine system, leading to reproductive disorders $^{7}$ (Environment Canada and Health Canada, 2012). For example, PFOA, which is quickly absorbed, can be transferred to the fetus through the placenta, and to infants via breast milk ${ }^{8}$.

Potential toxicity events were explored using the POP-PPAN model. First, data were compiled from the CompTox database (as of July 18,2019$)$. Considering the cytotoxicity threshold (5.35 $\mu \mathrm{M})$, nine proteins were extracted (Tab. S6 ${ }^{4}$ ). Using the POP-PPAN model, eight proteins were added, allowing the formation of a protein complex of 17 proteins linked to PFOA (Tab. S6 ${ }^{4}$ ) that contains 8 protein-protein associations. Only 3 AOPs (related to hepatic steatosis, breast cancer, and fetal testis) were identified via 3 targets, i.e., NR1I2, ESR1 and NR3C1.

ORA studies were performed on the diverse data sources. Among the 17 proteins involved in the complex linked to PFOA, two were not mapped (CYP2A2 and CYP2C11), meaning that these proteins are not yet annotated in the databases. The results of the GO terms investigation showed that the 15 proteins are classified in the metabolic process category (Tab. S $7^{4}$ ). Among them, 8 are assigned to the developmental process and 4 to growth and reproductive processes. The KEGG pathway enrichment analysis did not reveal any significant result, meaning that no pathway was highly connected to PFOA. By exploring the non-significant associations, several metabolism-related pathways could be reported as "drug metabolism" (Tab. S74). The enrichment analysis with the Reactome database identified 6 significant pathways, the most significant being the nuclear receptor transcription pathway (FDR of 2.40 ${ }^{\mathrm{e}-8}$ via ESR1; NR1I2; NR3C1; RARB; RORA and VDR). Interestingly, the circadian clock, which is part of regulatory pathways, was also identified (FDR $2.20^{\mathrm{e}-2}$ via NR3C1; PPP1CA; RORA). No diseases were associated after enrichment of the protein complex using the DisGeNET database due to the small size of the protein complex. More knowledge and data integration are necessary in order to predict toxicity of such compounds. Looking at other existing databases such as the Comparative Toxicogenomics Database (CTD), PFOA was found to be connected to many more proteins, but not necessarily directly, and no cytotoxicity data were found (Davis et al., 2017). For instance, the biological target PPARA is highly associated with PFOA (in CTD as of September 8, 2019), via interactions such as "PPARA protein inhibits the reaction PFOA results in decreased expression of ADH7 mRNA" (Rosen et al., 2008), which is very difficult to integrate into a toxicological model.

\subsubsection{Global mapping of the 28 sPOPs in biological and disease spaces}

Potential toxicities were explored using the POP-PPAN model for the other 26 listed sPOPs using the approach described above for dicofol and PFOA. First, sPOP-protein data were compiled from the CompTox database (as of November 2019), including the cytotoxicity thresholds (Tab. 2). Among the $28 \mathrm{sPOPs}$, no data was retrieved for three compounds, i.e., PCB 118, dechlorane plus, and perfluorohexane sulfonic acid (PFHxS). Three other chemicals did not pass the cytotoxicity threshold (BDE 47, BDE 99 and BDE 153), and fewer than 5 proteins were identified for 11 compounds. Therefore, only 11 sPOPs could undergo the systems toxicology analyses. Using the POP-PPAN model, first order interactor proteins were added individually to the 11 sPOP-proteins to form protein complexes (Tab. 2), and ORA analyses were performed.

In the $\mathrm{GO}$ analyses, the main biological processes involving proteins from the different identified protein complexes were the metabolic, the developmental and the reproductive processes. From a pathway point of view, based on the KEGG ORA, var-

\footnotetext{
7 https://www.ec.gc.ca/ese-ees/370AB133-3972-454F-A03A-F18890B58277/PFOA_EN.pdf

8 https://echa.europa.eu/documents/10162/8059e342-1092-410f-bd85-80118a5526f5
} 


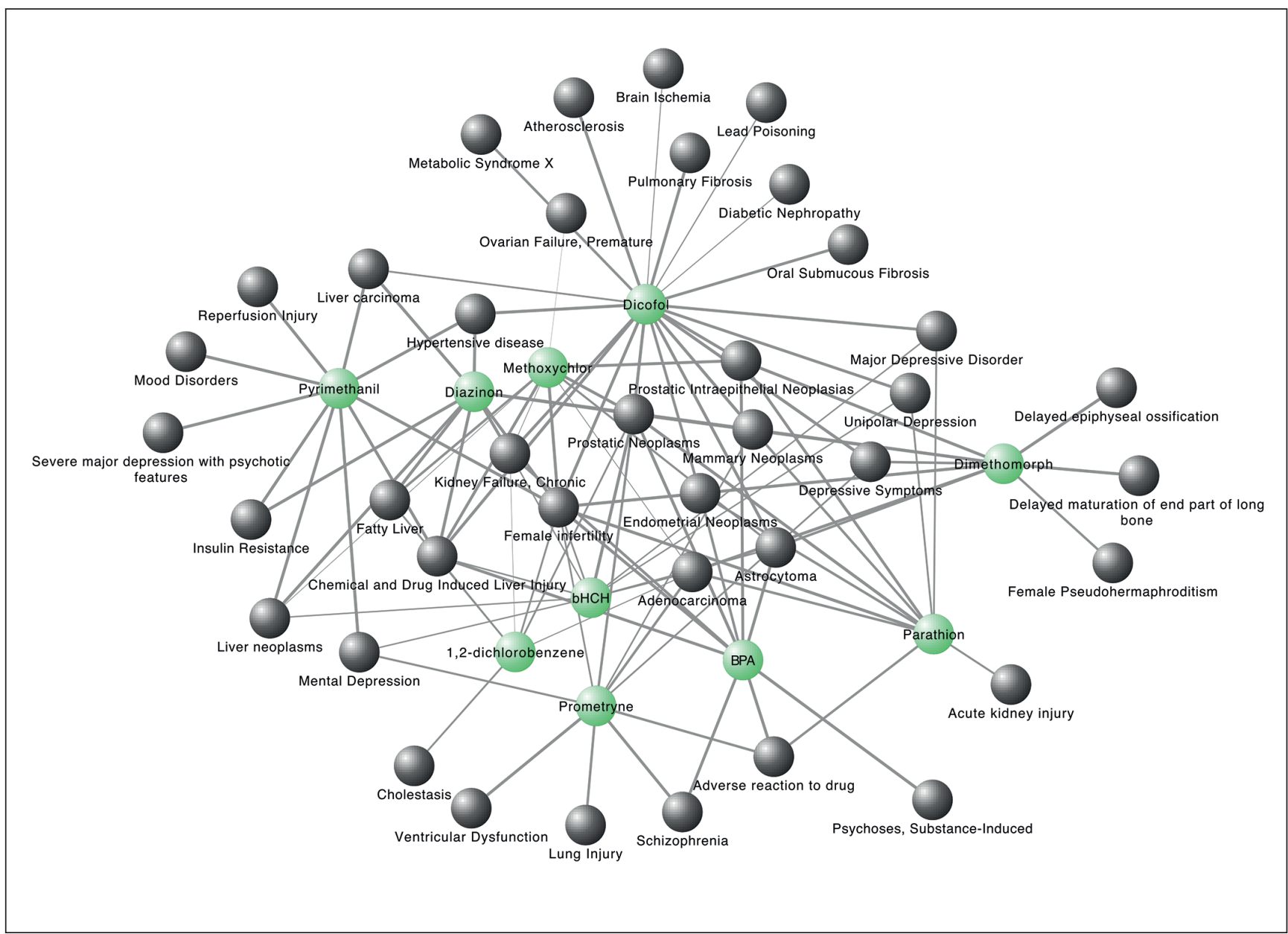

Fig. 5: Mapping of 10 POP candidates in the disease space

Each green node represents a chemical, and each grey node a disease. Linkages between chemicals and disorders were obtained by an over-representation analysis using the DisGeNet database on the protein complexes associated to each chemical that were identified using the developed POP-protein network. The width of the edges is according to statistical significance.

ious metabolism pathways were retrieved as statistically significant, such as linoleic acid, retinol, arachidonic acid or tryptophan metabolism. Within the Reactome enrichment, mainly lipid metabolism-related pathways were identified. The last analysis was performed using the DisGeNet database in order to decipher linkage between the sPOPs and diseases. Two major systems appear to be affected by 10 of these candidate POPs (no statistical significance was found for PFOA), i.e., the nervous system and reproductive system (Fig. 5). Such findings are well in line with previous studies. A recent review highlighted the pivotal role of lipids in neuronal functions, emphasizing the potential use of lipids as biomarkers for major depressive disorders (Parekh et al., 2017). Our model allowed us to identify which sPOPs (dicofol, parathion, beta-hexachlorocyclohexane $(\beta-\mathrm{HCH}))$ may be associated with major depressive disorders as well as other neural diseases. For example, to date, among the 405 available publications on dicofol, none mention a link between dicofol and major depression disorder (PubMed, as of December 09, 2019).
Seven chemicals were statistically associated with the reproductive disorder female infertility $\left(2.56^{\mathrm{e}-06}<\right.$ FDR $\left.<7.98^{\mathrm{e}-04}\right)$. Some links between the reproductive system and sPOPs are already known (methoxychlor), others have not been characterized yet (diazinon, dimethomorph, pyrimethanil) (PubMed, as of December 9, 2019).

\section{Discussion}

The proposed computational network-based model is different and complementary to existing molecular modelling approaches such as quantitative structure activity relationships (QSAR) and quantitative structure property relationships (QSPR) that are based on structural information of chemicals. For example, efficient QSAR and QSPR models have been developed to predict, in a quantitative manner, eco-toxicological, half-life or chemical properties of environmental interest that can be used for regula- 
tory purposes and in order to design alternative chemicals that are safer for the environment (Khan et al., 2019a,b; Mansouri et al., 2018). Another strategy is the use of the read-across approach to develop predictive models to derive reference points for hazard characterization of untested chemicals from the available experimental data for structurally-similar compounds (Benfenati et al., 2019). A network-based model has the advantage of being independent of the chemical's structural information and can be used to propose a hypothesis for toxicological profiling of chemical substances. Such systems modeling methods combine various types of information, including chemical-protein interactions, protein-protein interactions, protein-disease annotations, chemical-adverse effect evidence or chemical exposure information (Knudsen et al., 2013; Kongsbak et al., 2014; Taboureau and Audouze, 2017). A network of complex diseases was developed by Gohlke et al. (2009) by integrating molecular pathways associated with both genetic and environmental factors.

In the presented approach, known POP-protein interactions were used to develop a selective and predictive POP-PPAN model to which diverse data types, such as AOP information, biological pathways, and protein-disease annotations, were integrated for biological interpretation. As results, the model identified, in a qualitative manner, putative links between candidate POP chemicals and mainly three adverse effect classes related to the reproductive, the nervous, and the metabolism systems.

Nevertheless, the development of such a NAM is still limited in terms of available data regarding chemical-protein interactions when taking into account the cytotoxicity levels. Indeed, only part of the available information was considered in order to create a more accurate and predictive model. An extension of the proposed model with integration of more data, including quantitative information such as dose levels, would be beneficial to fully evaluate the potential toxicity of emerging chemicals, including POPs, on human health.

Furthermore, one must take into consideration the so-called "Matthew effect", which describes the bias, in terms of available documentation and scientific literature, between well investigated chemicals and less well studied environmental compounds. This may impact computational models that are based on existing information, and therefore the potential innovation and discovery in research (Grandjean et al., 2011). Hence, the lack of association between a chemical and a biological event/disease may be the result of less available data used to create a model, rather than the lack of effect. Nevertheless, studies such as the one proposed here, highlight the chemicals that need further attention and more testing. To address the need for new data, increasing efforts based on alternative test systems are on-going. New technologies, including the continued expansion of HTS (e.g., Tox21) and multi-omics (e.g., toxicogenomics) allow the production of large amounts of data (Richard et al., 2016).

Another important issue to take into consideration when working with multi-data integration, is the harmonization and the availability of the data. To generate relevant and predictive models, the interoperability of the data based on the goals established by findable, accessible, interoperable and reusable (FAIR) data principles, is essential to support computational toxicology and chemical safety evaluation (Watford et al., 2019).
With the advances in data generation and bioinformatics technology, more heterogeneous data will be publicly accessible and could be integrated, such as qualitative and quantitative information and know-how from animal, clinical, epidemiological, exposure and biomonitoring studies, allowing the development of emerging approaches to help understand the effects of POPs on human health. The challenge will be to map and integrate all these data into a common platform (Pawar et al., 2019).

The use of innovative informatics technology is also useful as this will allow the identification, in a systemic manner, of already known and published information. For example, a novel tool based on artificial intelligence, AOP-helpFinder, uses text mining to screen abstracts of published articles to identify co-mentioned terms, which could be a chemical and an AOP event (Carvaillo et al., 2019; Rugard et al., 2019). Key information can also be identified by integrating multiple data sources from HTS studies by using frequent itemset mining (FIM) to develop a computational predictive model (Oki and Edwards, 2016).

\section{Conclusion}

To support chemical safety assessment, there is a need to develop and apply novel innovative and validated methods that are animal-free approaches in order to decipher the toxicological profiling of chemical substances such as POPs. While in silico testing cannot substitute for in vitro or in vivo testing, it can help focus on particular substances and targets to allow priority-setting for more efficient testing that can greatly help early risk assessment of POPs.

\section{References}

Andersen, M. E., McMullen, P. D., Phillips, M. B. et al. (2019). Developing context appropriate toxicity testing approaches using new alternative methods (NAM). ALTEX 36, 523-534. doi:10.14573/altex.1906261

Ashburner, M., Ball, C. A., Blake, J. A. et al. (2000). Gene ontology: Tool for the unification of biology. The Gene Ontology Consortium. Nat Genet 25, 25-29. doi:10.1038/75556

Audouze, K., Juncker, A. S., Roque, F. J. et al. (2010). Deciphering diseases and biological targets for environmental chemicals using toxicogenomics networks. PLoS Comput Biol 6, e1000788. doi:10.1371/journal.pcbi.1000788

Audouze, K. and Grandjean, P. (2011). Application of computational systems biology to explore environmental toxicity hazards.Environ HealthPerspect 119, 1754-1759. doi:10.1289/ehp. 1103533

Audouze, K., Brunak, S. and Grandjean, P. (2013). A computational approach to chemical etiologies of diabetes. Sci Rep 3, 2712. doi:10.1038/srep02712

Audouze, K., Tromelin, A., Le Bon, A. M. et al. (2014). Identification of odorant-receptor interactions by global mapping of the human odorome. PLoS One 9, e93037. doi:10.1371/journal. pone.0093037

Benfenati, E., Chaudhry, Q., Gini, G. et al. (2019). Integrating in silico models and read-across methods for predicting toxicity 
of chemicals: A step-wise strategy. Environ Int 131, 105060. doi:10.1016/j.envint.2019.105060

Carvaillo, J.-C., Barouki, R., Coumoul, X. et al. (2019). Linking bisphenol $\mathrm{S}$ to adverse outcome pathways using a combined text mining and systems biology approach. Environ Health Perspect 127, 47005. doi:10.1289/EHP4200

Chihara, M., Yoshihara, K., Ishiguro, T. et al. (2016). Association of NR3C1/glucocorticoid receptor gene SNP with azoospermia in Japanese men. J Obstet Gynaecol Res 42, 59-66. doi:10.1111/jog. 12877

Corton, J. C., Kleinstreuer, N. C. and Judson, R. S. (2019). Identification of potential endocrine disrupting chemicals using gene expression biomarkers. Toxicol Appl Pharmacol 380, 114683. doi:10.1016/j.taap.2019.114683

Davis, A. P., Grondin, C. J., Johnson, R. J. et al. (2017). The comparative toxicogenomics database: Update 2017. Nucleic Acids Res 45, D972-D978. doi:10.1093/nar/gkw838

de Lichtenberg, U., Jensen, L. J., Brunak, S. et al. (2005). Dynamic complex formation during the yeast cell cycle. Science 307, 724-727. doi:10.1126/science. 1105103

Deng, P., Li, X., Petriello, M. C. et al. (2019). Application of metabolomics to characterize environmental pollutant toxicity and disease risks. Rev Environ Health 34, 251-259. doi: 10.1515/reveh-2019-0030

Fabregat, A., Jupe, S., Matthews, L. et al. (2018). The reactome pathway knowledgebase. Nucleic Acids Res 46, D649-D655. doi:10.1093/nar/gkx1132

Gohlke, J. M., Thomas, R., Zhang, Y. et al. (2009). Genetic and environmental pathways to complex diseases. BMC Syst Biol 3, 46. doi:10.1186/1752-0509-3-46

Grandjean, P., Eriksen, M. L., Ellegaard, O. et al. (2011). The Matthew effect in environmental science publication: A bibliometric analysis of chemical substances in journal articles. Environ Health 10, 96. doi:10.1186/1476-069X-10-96

Hartung, T., FitzGerald, R. E., Jennings, P. et al. (2017). Systems toxicology: Real world applications and opportunities. Chem Res Toxicol 30, 870-882. doi:10.1021/acs.chemrestox. $7 \mathrm{~b} 00003$

Haug, L. S., Thomsen, C., Brantsaeter, A. L. et al. (2010). Diet and particularly seafood are major sources of perfluorinated compounds in humans. Environ Int 36, 772-778. doi:10.1016/j. envint.2010.05.016

Haug, L. S., Huber, S., Becher, G. et al. (2011). Characterisation of human exposure pathways to perfluorinated compounds Comparing exposure estimates with biomarkers of exposure. Environ Int 37, 687-693. doi:10.1016/j.envint.2011.01.011

Judson, R., Houck, K., Martin, M. et al. (2016). Analysis of the effects of cell stress and cytotoxicity on in vitro assay activity across a diverse chemical and assay space. Toxicol Sci 153, 409. doi:10.1093/toxsci/kfw148

Kanehisa, M., Sato, Y., Furumichi, M. et al. (2019). New approach for understanding genome variations in KEGG. Nucleic Acids Res 47, D590-D595. doi:10.1093/nar/gky962

Kavlock, R., Chandler, K., Houck, K. et al. (2012). Update on EPA's ToxCast program: Providing high throughput decision support tools for chemical risk management. Chem Res Toxicol 25, 1287-1302. doi:10.1021/tx3000939
Kay, A. M., Simpson, C. L., Stewart, J. A. et al. (2016). The role of AGE/RAGE signaling in diabetes-mediated vascular calcification. J Diabetes Res 2016, 6809703. doi:10.1155/ 2016/6809703

Kelly, B. C., Ikonomou, M. G., Blair, J. D. et al. (2007). Food web-specific biomagnification of persistent organic pollutants. Science 317, 236-239. doi:10.1126/science.1138275

Khan, K., Baderna, D., Cappelli, C. et al. (2019a). Ecotoxicological QSAR modeling of organic compounds against fish: Application of fragment based descriptors in feature analysis. Aquat Toxicol 212, 162-174. doi:10.1016/j.aquatox.2019.05.011

Khan, P. M., Baderna, D., Lombardo, A. et al. (2019b). Chemometric modeling to predict air half-life of persistent organic pollutants (POPs). J Hazard Mater 382, 121035. doi:10.1016/j. jhazmat.2019.121035

Kleensang, A., Maertens, A., Rosenberg, M. et al. (2014). Pathways of toxicity. ALTEX 31, 53-61. doi:10.14573/altex. 1309261

Knudsen, T., Martin, M., Chandler, K. et al. (2013). Predictive models and computational toxicology. Methods Mol Biol 947, 343-374. doi:10.1007/978-1-62703-131-8_26

Kongsbak, K., Vinggaard, A. M., Hadrup, N. et al. (2014). A computational approach to mechanistic and predictive toxicology of pesticides. ALTEX 31, 11-22. doi:10.14573/altex. 1304241

Koual, M., Cano-Sancho, G., Bats, A.-S. et al. (2019). Associations between persistent organic pollutants and risk of breast cancer metastasis. Environ Int 132, 105028. doi:10.1016/j. envint.2019.105028

Krewski, D., Acosta, D., Jr., Andersen, M. et al. (2010). Toxicity testing in the $21^{\text {st }}$ century: A vision and a strategy. $J$ Toxicol Environ Health B 13, 51-138. doi:10.1080/10937404.2010.48 3176

Liu, Y. and Liu, R. (2012). The interaction of $\alpha$-chymotrypsin with one persistent organic pollutant (dicofol): Spectroscope and molecular modeling identification. Food Chem Toxicol 50, 3298-3305. doi:10.1016/j.fct.2012.06.037

MacLellan, K. N., Bird, D. M., Fry, D. M. et al. (1996). Reproductive and morphological effects of o,p'-dicofol on two generations of captive American kestrels. Arch Environ Contam Toxicol 30, 364-372. doi:10.1007/bf00212295

Mansouri, K., Grulke, C. M., Judson, R. S. et al. (2018). OPERA models for predicting physicochemical properties and environmental fate endpoints. $J$ Cheminform 10, 10. doi:10.1186/ s13321-018-0263-1

Naderi, M., Wong, M. Y. L. and Gholami, F. (2014). Developmental exposure of zebrafish (Danio rerio) to bisphenol-S impairs subsequent reproduction potential and hormonal balance in adults. Aquat Toxicol 148, 195-203. doi:10.1016/j.aquatox. 2014.01.009

Nordkap, L., Almstrup, K., Nielsen, J. E. et al. (2017). Possible involvement of the glucocorticoid receptor (NR3C1) and selected NR3C1 gene variants in regulation of human testicular function. Andrology 5, 1105-1114. doi:10.1111/andr.12418

NRC - National Research Council (2007). Toxicity Testing in the $21^{\text {st }}$ Century: A Vision and a Strategy. Washington, DC, USA: The National Academies Press. doi:10.17226/11970 
Oki, N. O. and Edwards, S. W. (2016). An integrative data mining approach to identifying adverse outcome pathway signatures. Toxicology 350-352, 49-61. doi:10.1016/j.tox.2016.04.004

Parekh, A., Smeeth, D., Milner, Y. et al. (2017). The role of lipid biomarkers in major depression. Healthcare (Basel) 5, 5. doi:10.3390/healthcare5010005

Pawar, G., Madden, J. C., Ebbrell, D. et al. (2019). In silico toxicology data resources to support read-across and (Q)SAR. Front Pharmacol 10, 561. doi:10.3389/fphar.2019.00561

Piñero, J., Bravo, À., Queralt-Rosinach, N. et al. (2017). DisGeNET: A comprehensive platform integrating information on human disease-associated genes and variants. Nucleic Acids Res 45, D833-D839. doi:10.1093/nar/gkw943

Richard, A. M., Judson, R. S., Houck, K. A. et al. (2016). ToxCast chemical landscape: Paving the road to $21^{\text {st }}$ century toxicology. Chem Res Toxicol 29, 1225-1251. doi:10.1021/acs. chemrestox.6b00135

Rosen, M. B., Abbott, B. D., Wolf, D. C. et al. (2008). Gene profiling in the livers of wild-type and PPARalpha-null mice exposed to perfluorooctanoic acid. Toxicol Pathol 36, 592-607. doi:10.1177/0192623308318208

Rugard, M., Courmoul, X., Carvaillo, J.-C. et al. (2019). Deciphering adverse outcome pathway network linked to bisphenol $\mathrm{F}$ using text mining and systems toxicology approaches. Toxicol Sci 173, 32-40. doi:10.1093/toxsci/kfz214

Smirnova, L., Kleinstreuer, N., Corvi, R. et al. (2018). 3S Systematic, systemic, and systems biology and toxicology. ALTEX 35, 139-162. doi:10.14573/altex.1804051

Taboureau, O. and Audouze, K. (2017). Human environmental disease network: A computational model to assess toxicology of contaminants. ALTEX 34, 289-300. doi:10.14573/altex. 1607201

Tarassov, K., Messier, V., Landry, C. R. et al. (2008). An in vivo map of the yeast protein interactome. Science 320, 1465-1470. doi: $10.1126 /$ science 1153878

The Gene Ontology Consortium (2019). The gene ontology resource: 20 years and still GOing strong. Nucleic Acids Res 47, D330-D338. doi:10.1093/nar/gky1055
Villeneuve, D. L., Crump, D., Garcia-Reyero, N. et al. (2014). Adverse outcome pathway (AOP) development I: Strategies and principles. Toxicol Sci 142, 312-320. doi:10.1093/toxsci/ kfu199

Vinggaard, A. M., Hnida, C., Breinholt, V. et al. (2000). Screening of selected pesticides for inhibition of CYP19 aromatase activity in vitro. Toxicol In Vitro 14, 227-234. doi:10.1016/ S0887-2333(00)00018-7

Watford, S., Edwards, S., Angrish, M. et al. (2019). Progress in data interoperability to support computational toxicology and chemical safety evaluation. Toxicol Appl Pharmacol 380, 114707. doi:10.1016/j.taap.2019.114707

Williams, A. J., Grulke, C. M., Edwards, J. et al. (2017). The CompTox chemistry dashboard: A community data resource for environmental chemistry. J Cheminform 9, 61. doi:10.1186/ s13321-017-0247-6

Yildirim, M. A., Goh, K.-I., Cusick, M. E. et al. (2007). Drug-target network. Nat Biotechnol 25, 1119-1126. doi:10.1038/ nbt1338

Yu, H., Braun, P., Yildirim, M. A. et al. (2008). High-quality binary protein interaction map of the yeast interactome network. Science 322, 104-110. doi:10.1126/science. 1158684

Zhong, G., Xie, Z., Cai, M. et al. (2012). Distribution and airsea exchange of current-use pesticides (CUPs) from East Asia to the high Arctic Ocean. Environ Sci Technol 46, 259-267. doi:10.1021/es202655k

\section{Conflict of interest}

The authors declare they have no actual or potential competing financial interests.

\section{Acknowledgments}

The authors would like to acknowledge the CREATIvE project. This project has received funding from the French National Research Agency (ANR-18-CE34-0001-01). This work was also supported by the Université de Paris and INSERM. 